\title{
Feasibility Study of Full-duplex Relaying in Satellite Networks
}

\author{
Bhavani Shankar Mysore R*, Gan Zheng ${ }^{\dagger}$, Sina Maleki*, and Björn Ottersten * \\ * Interdisciplinary Centre for Security, Reliability and Trust, University of Luxembourg, L-2721 Luxembourg \\ $\dagger$ School of Computer Science and Electronic Engineering, University of Essex, Colchester, UK, CO4 3SQ \\ Email: bhavani.shankar@uni.lu, ganzheng@essex.ac.uk, sina.maleki@uni.lu bjorn.ottersten@uni.lu
}

\begin{abstract}
With the successful implementation of full-duplex radio prototypes, traditional orthogonal half-duplex communications is deemed to be inefficient in certain terrestrial applications. While full-duplex techniques are gaining interest in terrestrial communications, thanks to the trend of short-distance and low-power transmissions, their application to satellite communications has drawn little attention. Motivated by this, the paper explores the use of the full-duplex relaying operation on-board the satellite in a DVB-S2 compliant network. Self-interference, whose management is the key component of a full-duplex communication, is the focus of study in this paper. Modelling the effects of self-interference and power amplifier nonlinearities on the quality of the received signal in undertaken. Subsequently, closed-form expressions for the various interference components are derived. The numerical evaluations of derived expressions rely on realistic link budgets and indicate substantial gains in spectral efficiency when self-interference can be well calibrated and measured. This confirms that the satellite full-duplex communications could be a promising solution for the efficient use of satellite spectrum, at least from a technical point of view.
\end{abstract}

\section{INTRODUCTION}

Thanks to their wide coverage, satellites serve as an effective fora to provide broadcast and interactive broadband services, especially to geographical areas under-served by terrestrial infrastructure. In a typical architecture, satellite acts as a relay for conveying information from the on-ground transmitter to an on-ground receiver. Towards satisfying an increased demand for high throughput from satellite networks, several techniques and technologies have been considered towards enhancing the spectral efficiency of satellite communications either by acquiring additional spectrum or better utilization of existing ones. A typical example for the former is the considered migration to $\mathrm{Q} / \mathrm{V}$ bands and optical communications. Use of additional spectrum is influenced by existing regulations and technological maturity of the component sub-systems. Better utilization of the existing spectrum is currently been pursued through enhancements to DVB-S2 standard [1], [2], multibeam architectures with associated flexibility [3], full frequency reuse [4] and cognitive techniques [5] amongst others.

Full-duplex communications, where a node receives and transmits simultaneously in the same frequency band, has received much attention recently for its potential to double the transmission rate or save half the bandwidth [6], [7], [8]. However, in reality, the ideal gains are reduced by the self-interference (SI) phenomenon arising due to leakage of signal power from the transmitted waveform onto the waveform being received [9]. Hence a key aspect towards incorporation of full-duplex paradigm involves an analysis of the tolerable self-interference levels followed by an implementation of compensation techniques to achieve the said interference level. The SI cancellation can be broadly categorized as passive cancellation and active cancellation [6]. Passive suppression is to isolate the transmit and receive antennas [10]. Active suppression is to exploit a node's knowledge of its own transmit signal to cancel the SI, which typically includes analog cancellation and digital cancellation [11]. In the recent work [12] and [13], the promising results show that the SI can be suppressed to the noise level in both single-antenna and multi-antenna cases using the 802.11ac standard. The effects of RF imperfections such as transmitter power amplifier nonlinear distortion as well as IQ mixer imbalances on the SI cancellation have been studied in terrestrial communications [14]. A particular full-duplex architecture having similarities to the traditional satellite communications is the full-duplex relaying. While a rich literature on the analysis and suppression of SI exists in terrestrial applications, a similar study is not available for satellite systems. Analog physical layer coding techniques saving bandwidth have been considered for specific satellite scenarios [15], [16]. However, the literature on network coding suggests improvements over the analog design, thereby warranting a study of full-duplex relaying in satellites.

In this paper, unlike [15], [16], we consider the use of in-band full-duplex relaying in satellite systems for the first time. A full duplex relaying operation is considered on-board the satellite where the uplink and the downlink transmissions occur simultaneously over the same frequency band. Clearly, incorporating full-duplexing allows for provisioning additional services at little or no extra cost and this can have a profound impact on all the actors in the satellite industry. However, adopting the full-duplex mode on the satellite faces several technical challenges, even without considering the regulatory restrictions. A key differentiator of the satellite systems from their terrestrial counterparts, apart from technology and complexity, is the distance traversed by the signal. The distances no longer correspond to the short-distances (up to a few $\mathrm{km}$ as in terrestrial small cells) for which all known applications of full-duplex radios are confined to. Hence, before discussing SI mitigation techniques and their implementation, an analysis of the allowable SI levels for which such a full-duplex operation provides gains over the traditional half-duplex operation is warranted. This motivates the work carried out in the paper.

In this work, we characterize, model and analyze the various components of SI in the context of in-band full-duplex relaying on-board the satellite. We then determine the power level of SI for which a fullduplex implementation provides gains over the traditional half-duplex for DVB-S2 transmissions. These values serve as benchmark for later implementation of on-board/ on-ground SI cancellation. Simulation results show that the end-to-end rate of the full-duplex operation greatly outperforms that of the conventional half-duplex mode when the resulting SI power is low. This illustrates the feasibility aspect of full-duplex satellite relaying from the technical point of view. In particular, we identify several unique opportunities which are not available in the terrestrial applications, but surprisingly favor the full-duplex satellite implementation. This paves way to assess the technological challenges of SI cancellation as a next step towards implementing such systems.

\section{Scenario And System Model}

In this section, we first describe the reference scenario involving traditional half-duplex transmission. Subsequently, the full-duplex mode is introduced. The models for the propagation channel as well as the transponder are then mentioned. Generation of self interference is then described leading to an end-to-end system model. 


\section{A. Reference Half-Duplex System Scenario}

We consider communications from a Gateway (GW) through the broadband Geostationary (GEO) satellite to an user terminal (UT) in the Ka-band with DVB-S2 compliant waveforms. Typical applications include voice and data connectivity, broadband internet and other interactive services. Several high throughput satellites supporting such services have been recently launched including Ka-Sat, ViaSat 1, etc. A bent-pipe satellite is typically employed whose functions comprise filtering, frequency translation and amplification. The GEO satellite as well as the UT further operate in the half-duplex mode with separate uplink and downlink frequency bands. As an example, in the Ka-Sat, the feeder up and downlinks are in the bands $28-29.5 \mathrm{GHz}$ and 18.4-19.7 GHz respectively; the user up and downlinks employ the bands $29.5-30 \mathrm{GHz}$ and $19.7-20.2 \mathrm{GHz}$ respectively. In this paper, we focus on the forward link from the GW to the UT. To subsume cases where the GW is not located in the coverage area, a dedicated antennas for the feeder and user links are assumed. Figure 1 presents a simplified model of the considered architecture.

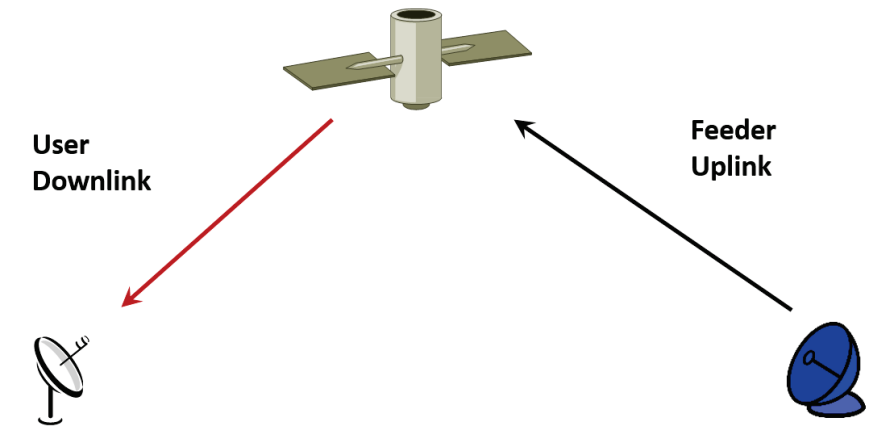

UserTerminal

Gateway

Fig. 1. Half-duplex transmission on the forward path : Both links use different frequencies

\section{B. Full-Duplex Relaying Scenario}

We now describe a system where the satellite operates in the fullduplex mode. In particular, referring to Fig. 2, the envisaged scenario involves the use of the same frequency band on the feeder uplink (GW to satellite) and user downlink (satellite to UT).The return path from UT to GW can also be implemented in a similar full-duplex manner. For this initial study, we further assume that forward and return paths are on different bands and it suffices to consider one of them.

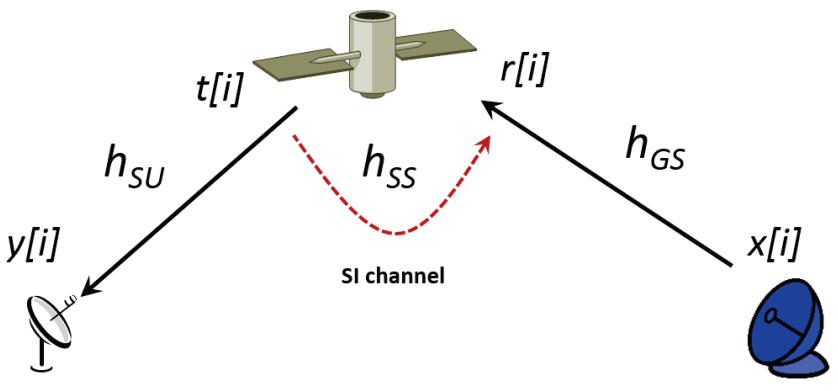

UserTerminal

Fig. 2. Full-duplex forward path relaying showing SI

The key aspects of the considered scenario are:
- In the ideal case, where a symmetric traffic distribution is assumed on all links, a gain of $100 \%$ in bandwidth is envisaged.

- The user terminal still operates in the half-duplex mode but the satellite operates in the full-duplex mode. This only requires minimal changes to legacy on-ground systems.

- The proposed full-duplex architecture can be construed as being straight-forward when the GW and UT are geographically separated. However, considering the low received power onboard the satellite, a rigourous analysis of the impact of selfinterference is needed to assess its feasibility.

The re-assignment of bands invokes regulatory aspects; however, for the current technical study, this important aspect is overlooked. However, the focus of the paper is on the analysis of SI; we begin the same with the modelling of associated components.

\section{Satellite Transponder Model}

To focus on the full-duplex aspects, we assume that the uplink and downlink are Additive White Gaussian Noise (AWGN) channels. Further, the transponder on the satellite comprises of the input multiplexing filter (IMUX), a high power amplifier (HPA) and the output multiplexing filter (OMUX). Typical IMUX and OMUX filter characteristics are obtained from [1]; these filters exhibit group-delay variations only at the edges and can be considered to impart linear phase component to the signal in the pass-band. On the other hand, the HPA is considered to be frequency non-selective while exhibiting a non-linear characteristic [1]. The typical transfer function of such a HPA is described through its AM/ AM and AM/ PM (AM: Amplitude Modulation, PM: Phase Modulation) characteristics [1]. In this work, we assume that the employed frequencies to lie within the passband of the IMUX and OMUX filters and that the HPA is driven in the quasi-linear region of operations. Finally, the full-duplex mode of operation results in SI from the user downlink signal onto the feeder uplink signal.

\section{Self Interference and System Model}

With the channel components and effects described, we now proceed with the modelling of the system in Fig. 2. While the uplink and downlink channels are AWGN, the transponder per-se is a weakly non-linear system without memory. Such non-linear systems can be modelled using the Volterra series [17]. While Volterra expansion is an infinite power series, exploiting the weak non-linearity, we truncate the expansion to the third degree and model the output $s_{\text {out }}[i]$ of the transponder as $s_{\text {out }}[i]=\alpha_{1} s_{\text {in }}[i]+\alpha_{3}\left|s_{\text {in }}[i]\right|^{2} s_{\text {in }}[i]$, where $s_{\text {in }}[i]$ is the transponder input and $\alpha_{1}, \alpha_{3}$ are the co-efficients corresponding the linear and third degree terms, respectively. Further, the SI is modelled as a delayed and scaled feedback of the transponder output. The resulting system can be modelled as,

$$
\begin{aligned}
r[i] & =h_{G S} x[i]+h_{S S} t[i-\tau]+n_{R}[i], \\
t[i] & =\beta\left[\alpha_{1} r[i]+\alpha_{3}|r[i]|^{2} r[i]\right]+n_{T}[i], \\
y[i] & =h_{S U} t[i]+n_{U}[i]
\end{aligned}
$$

where, $x[i]$ is the signal from the GW at instance $i, h_{G S}$ models the gain on uplink, $n_{R}[i]$ is the noise at the front end of the receiving antenna on-board the satellite and $r[i]$ is the resulting on-board received signal. Equation (2) indicates the transponder processing

Gateway incorporating the HPA non-linearity (based on truncated Volterra series) and transmitter noise. The scaling factor $\beta$ is to ensure that the power transmitted from the satellite remains within the prescribed bounds. For ease of analysis, we model the SI component as a delayed (by $\tau$ ) and scaled version of $t[i]$, the scaling being 
governed by $h_{S S}$. The factor $h_{S S}$ determines the strength of the SI; it can also be construed as the residual SI channel after certain SI cancellation measures are applied. Further, the signal received onground is depicted using $y[i]$ with $n_{U}[i]$ being the receiver noise and $h_{S U}$ denoting the downlink channel gain.

Focussing on the line-of-sight communication, we model the channel gains as $h_{G S}=\gamma_{G S} G_{G S} d_{G S}^{L_{G S}}$ and $h_{S U}=\gamma_{S U} G_{S U} d_{S U}^{L_{S U}}$, where the variables $G, d, L$ and $\gamma$ denote antenna gain (including both transmit and receive), distance, path loss exponent and the pathloss scaling factor, respectively. The noise components $n_{R}[i], n_{T}[i]$ and $n_{U}[i]$ are modelled as i.i.d white Gaussian noise components with variance $\sigma_{R}^{2}, \sigma_{T}^{2}$ and $\sigma_{U}^{2}$ respectively.

\section{Interference AnALYsis And Mitigation}

In this section, we analytically determine the contribution of various impairments, especially of the SI, on the received Signal-toInterference-plus-Noise Ratio (SINR) based on the models in (1)-(3). While full-duplex mode can utilize larger bandwidth to enhance throughput, its SINR is reduced compared to the half-duplex mode due to SI. Thus, an end-to-end analysis is needed to ascertain gains of full-duplex communications. Further, the analysis can also serve as benchmark on interference cancellation algorithms with regards to their residual error levels. Elements relating to interference mitigation are discussed later in the section.

\section{A. SINR Analysis}

A first step in the analysis involves determining an expression for $t[i]$. While it is possible to obtain a recursion for $t[i]$ by substituting (1) in (2), the resulting expressions involve cross-products of $t[\cdot], x[\cdot], n_{R}[\cdot]$ and are intractable. Towards pursuing further analysis, we need to devise a tractable model relating $t[i]$ and $x[k]$ that (i) incorporates non-linearities, (ii) includes memory effects, if any and (iii) includes transmit and receiver noise. With these requirements in mind, we consider the simplified model given in (4) at the top of the next page. This recursion leads to (5) at the top of the next page. From (3) - (5), the following components can be identified easily,

- Desired signal : Obtained by scaling and rotating $x[i]$,

$$
I_{d e s}=\alpha_{1} \beta h_{S U} h_{G S} x[i] .
$$

- Non-linear component: This is due to the transponder characteristic modelled as a non-linear function without memory. This term is independent of the SI.

$$
I_{N L}=\alpha_{3} \beta h_{S U}\left|h_{G S}\right|^{2} h_{G S}|x[i]|^{2} x[i]
$$

- Self Interference, $I_{S I}$ : This comprises both linear and nonlinear terms and essentially has a wide time span induced by $h_{S S}$. These terms are given by,

$$
\begin{aligned}
I_{S I} & =\beta h_{S U} \sum_{l=1}^{\infty}\left(\alpha_{1} \beta h_{S S}\right)^{l}\left\{\alpha_{1} h_{G S} x[i-l \tau]\right. \\
& \left.+\alpha_{3}\left|h_{G S}\right|^{2} h_{G S}|x[i-l \tau]|^{2} x[i-l \tau]\right\} .
\end{aligned}
$$

- Uplink Noise, $I_{\eta}$ : The noise component $n_{R}[i]$ is generated by the transponder and the resulting noise, unaffected by SI, is

$$
I_{\eta}=\beta h_{S U}\left[\alpha_{1} n_{R}[i]+\alpha_{3}\left|n_{R}[i]\right|^{2} n_{R}[i]\right]
$$

- Downlink and Receiver noise, $I_{\nu}$ : This component arises due to $n_{T}[i]$ and $n_{U}[i]$ and is independent of the SI. In particular,

$$
I_{\nu}=h_{S U} n_{T}[i]+n_{U}[i]
$$

- Full-Duplexing Noise, $I_{\gamma}$ : This term arises due to the SI phenomenon; note that the transmit and receive noise sources on-board the satellite $\left(n_{R}[\cdot], n_{T}[\cdot]\right)$ are included.

$$
\begin{aligned}
I_{\gamma} & =h_{S U} \sum_{l=1}^{\infty}\left(\alpha_{1} \beta h_{S S}\right)^{l}\left\{\alpha_{1} \beta n_{R}[i-l \tau]\right. \\
& \left.+\alpha_{3} \beta\left|n_{R}[i-l \tau]\right|^{2} n_{R}[i-l \tau]+n_{T}[i-l \tau]\right\}
\end{aligned}
$$

Assuming that the quantities $h_{G S}, h_{S S}, h_{S U}, \alpha_{k}$ are known at the receiver through prior calibration, we obtain the resulting SINR as,

$$
S I N R \triangleq \frac{E\left[\left|I_{\text {des }}\right|^{2}\right]}{E\left[\left|y[i]-I_{\text {des }}\right|^{2}\right]} \quad \text { where. }
$$

$$
\begin{aligned}
E\left[\left|I_{\text {des }}\right|^{2}\right]= & \left|\alpha_{1} \beta h_{S U} h_{G S}\right|^{2} E\left[|x[i]|^{2}\right], \\
E\left[\left|I_{N L}\right|^{2}\right]= & \left|\alpha_{3} \beta h_{S U}\right|^{2}\left|h_{G S}\right|^{6} E\left[|x[i]|^{6}\right], \\
E\left[\left|I_{S I}\right|^{2}\right]= & \left|\beta h_{S U}\right|^{2} \frac{\left|\alpha_{1} \beta h_{S S}\right|^{2}\left|h_{G S}\right|^{2}}{1-\left|\alpha_{1} \beta h_{S S}\right|^{2}} \times\left[\left|\alpha_{1}\right|^{2} E\left[|x[i]|^{2}\right]\right. \\
& \left.+\left|h_{G S}\right|^{4}\left|\alpha_{3}\right|^{2} E\left[|x[i]|^{6}\right]+2 E\left[|x[i]|^{4}\right] \mathcal{R}\left(\left[\alpha_{1}\right]^{*} \alpha_{3}\right)\right], \\
E\left[\left|I_{\eta}\right|^{2}\right]= & \left|\beta h_{S U}\right|^{2}\left[\left|\alpha_{1}\right|^{2} \sigma_{R}^{2}+\left|\alpha_{3}\right|^{2} E\left[\left|n_{R}[i]\right|^{6}\right]\right. \\
& \left.+2 E\left[\left|n_{R}[i]\right|^{4}\right] \mathcal{R}\left(\left[\alpha_{1}\right]^{*} \alpha_{3}\right)\right], \\
E\left[\left|I_{\gamma}\right|^{2}\right]= & \frac{\left|\alpha_{1} \beta h_{S S}\right|^{2}}{1-\left|\alpha_{1} \beta h_{S S}\right|^{2}}\left(E\left[\left|I_{\eta}\right|^{2}\right]+\sigma_{T}^{2}\right), \\
E\left[\left|I_{\nu}\right|^{2}\right]= & \left|h_{S U}\right|^{2} \sigma_{T}^{2}+\sigma_{U}^{2},
\end{aligned}
$$

where $\mathcal{R}(\cdot)$ denotes the real part of the argument and $E[\cdot]$ is the Expectation operator. Evaluation of the SINR expressions require fourth and sixth order moments of the signal and noise components. Since $n_{R}[i]$ is Gaussian, it is possible to obtain these moments in terms of $\sigma_{R}^{2}$, for example $E\left[\left|n_{R}[i]\right|^{4}\right]=3 \sigma_{R}^{2}$. On the other hand, the signal is not known apriori; in fact, the constellation and the coding scheme will be decided based on the SINR conditions. To evolve out of this conundrum, we resort to bounds on $E\left[|x[i]|^{4}\right]$ and $E\left[|x[i]|^{6}\right]$. In particular, we evaluate these terms over the DVB-S2 constellations and choose the one that yields a higher interference (and a lower bound on SINR). Towards this, we see that $\left(E\left[|x[i]|^{4}\right], E\left[|x[i]|^{6}\right]\right)=$ $(1,1)$ for QPSK and 8 PSK, $(1.25,1.61)$ for 16 APSK and $(1.4,2.2)$ for 32 APSK, with $E\left[|x[i]|^{2}\right]=1$ for all. Thus a lower bound on SINR is obtained by employing the values derived from 32 APSK.

\section{B. Interference Mitigation}

The aforementioned analysis determines the contribution of the various interference components, HPA nonlinearities, on-board noise, SI and interactions thereof. A number of existing works have considered mechanisms to mitigate these impairments in the terrestrial scenario using RF cancellation followed by a digital cancellation [11], [12], [14]. In the pursued scenario, these techniques need to be used on-board the satellite. In the case of transparent satellites, fixed RF cancellation can be implemented on-board, while lack of processing capabilities preclude on-board digital cancellation. In particular, the SI channel component and the transmit noise are dealt with by fixed (non-adaptive) analog cancellation, e.g., tapping a line from the transmit antenna to the receive antenna. Assuming the true, timeinvariant, SI channel to be $\widetilde{h}_{S S}$, and the estimated SI channel to be $\widehat{h}_{S S}$, the cancellation signal can be produced by directly tapping a signal from the transmit antenna and multiplying it by $-\widehat{h}_{S S}$. Then the equivalent SI channel becomes,

$$
h_{S S}=\widetilde{h}_{S S}-\widehat{h}_{S S} .
$$

On ground techniques like predistortion [18] at the GW and equalization at the UT [19] need to be used to augment the on-board 


$$
\begin{aligned}
& t[i] \approx \alpha_{1} \beta\left[h_{S S} t[i-\tau]+h_{G S} x[i]+n_{R}[i]\right]+\alpha_{3} \beta\left[|x[i]|^{2} x[i]+\left|n_{R}[i]\right|^{2} n_{R}[i]\right]+n_{T}[i] . \\
& t[i] \approx \sum_{l=0}^{\infty}\left(\alpha_{1} \beta h_{S S}\right)^{l}\left[\beta\left\{\alpha_{1} h_{G S} x[i-l \tau]+\alpha_{1} n_{R}[i-l \tau]+\alpha_{3}\left|h_{G S}\right|^{2} h_{G S}|x[i-l \tau]|^{2} x[i-l \tau]+\alpha_{3}\left|n_{R}[i-l \tau]\right|^{2} n_{R}[i-l \tau]\right\}+n_{T}[i-l \tau]\right](5)
\end{aligned}
$$

TABLE I

PROPAGATION AND LINK BUDGET ASSUMPTIONS

\begin{tabular}{c|c}
\hline Ka-band & Value \\
\hline Feeder link & $28.5 \mathrm{GHz}$ \\
User link & $20 \mathrm{GHz}$ \\
GEO slot & $9^{\circ} \mathrm{E}$ \\
Bandwidth & $10 \mathrm{MHz}$ \\
Ground Station EIRP & $82 \mathrm{dBW}$ \\
Satellite EIRP & $60 \mathrm{dBW}$ \\
Free space loss & $213 \mathrm{~dB}$ \\
$\frac{G}{T}$ satellite & $20 \mathrm{~dB} / \mathrm{K}$ \\
$\frac{G}{T}$ user terminal & $15 \mathrm{~dB} / \mathrm{K}$ \\
\hline
\end{tabular}

analog interference mitigation. Predistortion tends to mitigate the non-linear distortions of the transponder [18] while a fractionally spaced equalizer with centroid decoding tends to mitigate residual non-linearity and group delay effects of the filters [19]. Thus, a hybrid interference cancellation approach needs to pursued for realizing fullduplex communications in transparent satellites.

On the other hand, there has been a trend in the satellite design towards including on-board digital processing. Currently, Digital Transparent Processing (DTP), which allows for limited digital processing with sampled waveforms, is being considered in a number of satellites (for e.g., SES16 by SES). Fully regenerative payloads, allowing data level processing, are envisaged for commercial communication satellites in the near future. With the onset of on-board processing, digital SI cancellation techniques can be readily implemented thereby bringing the envisaged full-duplex system closer to reality.

\section{Simulation Results}

In this section, we evaluate the feasibility of employing fullduplex mode in a representative satellite system. Towards this, we choose communication between a $9.2 \mathrm{~m} \mathrm{GW}$ station from GD Satcom and a $65 \mathrm{~cm}$ UT dish (Intellian GX65) ${ }^{1}$. For such a system, Table I provides the typical parameters, Under these conditions, matching the power predicted at the satellite by the link budget calculation with that obtained from (1) leads to an uplink channel gain of $h_{G S}=\sqrt{10^{\frac{-100}{10}}}$. Further, the noise $n_{R}[i]$ has a variance $\sigma_{R}^{2}=\sqrt{10^{\frac{-130}{10}}}$ (obtained from representative noise figures for a Ka-band satellite). Further, assuming the satellite power to be about 25 Watts, the parameters for the downlink, $h_{S U}, \sigma_{U}^{2}$ are obtained by solving the link-budget equations. While representative noise figures for the considered terminal yields $\sigma_{U}^{2}=\sqrt{10^{\frac{-135}{10}}}, h_{S U}$ will be dependent on the obtained Output Backoff (OBO). The parameters, $\alpha_{1}, \alpha_{3}$ are obtained through the standard indirect estimation of a linearized HPA whose characteristics are illustrated in [1]. Unless mentioned otherwise, the identification is performed at different Input Backoffs (IBOs) with 32 APSK modulation. The highest modulation of DVB-S2 is chosen so that the resulting HPA parameter values can be utilized for other lower order modulations as well. We choose

\footnotetext{
${ }^{1}$ Product details are available at www.gdsatcom.com and www.intelliantech.com
}

$\sigma_{T}^{2}=0.1 \sigma_{R}^{2} ; \beta$ is ideally set to ensure $E\left[|t[i]|^{2}\right]=P_{S}$, where $P_{S}$ is the satellite output power. Since the expression of $E\left[|t[i]|^{2}\right]$ is rather unwieldy, we resort to its simplification assuming $\alpha_{3}=0$ (neglecting non-linearities). After some manipulations, we obtain, $\beta=\sqrt{\frac{P_{S}-\left|h_{G S}\right|^{2}\left|h_{S S}\right|^{2} \sigma_{T}^{2}}{\left|\alpha_{1}\right|^{2}\left(\left|h_{G S}\right|^{2} E\left[|x[i]|^{2}\right]+\sigma_{R}^{2}+P_{S}\left|h_{S S}\right|^{2}\right)}}$, with $P_{S}=25 \mathrm{~W}$ in the current exercise.

Figure 3 relates the SINR values obtained by numerical evaluation of (6) and those obtained from exhaustive simulations. In these simulations, SINR is evaluated for the possible modulations (QPSK, 8PSK, 16APSK, 32 APSK) and different values of $\left|\frac{h_{S S}}{h_{G S}}\right|$ by implementing (1)-(3). Further, in each simulation, the amplifier coefficients are identified for the chosen IBO value using the target modulation. While the SINR curves for all modulations are presented in Fig. 3 (those for QPSK and 16 APSK are nearly identical), a system would use the modulations appropriately towards maximizing the spectral efficiency. The numerical evaluation of the SINR expression indeed provides a conservative representation of the SINR that would possibly be achieved by adapting modulation. Thus the numerical evaluations provide a faster means to obtain benchmark performance without resorting to exhaustive simulations involving a number of modulations. Henceforth, we focus on this methodology.

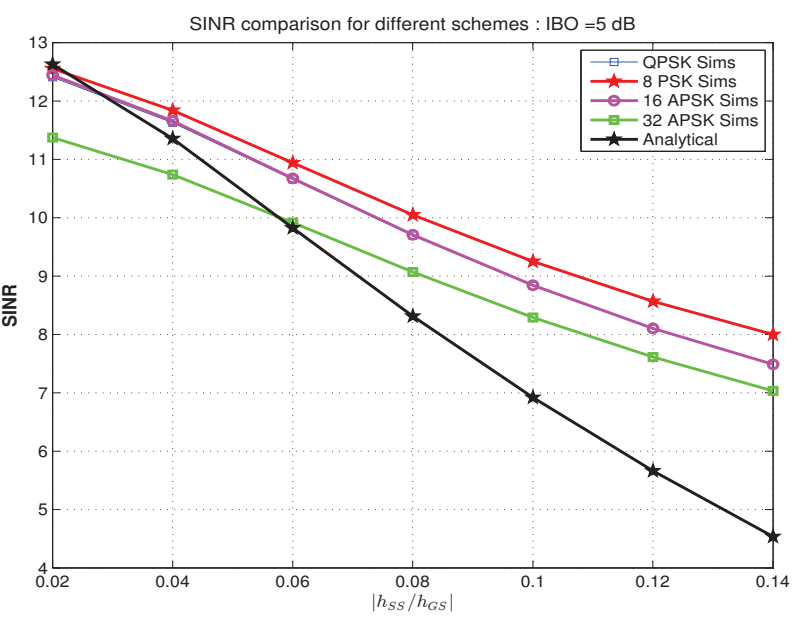

Fig. 3. Evaluation of SINR by different means : through analysis and through exhaustive simulations.

Figure 4 discusses the contribution of the various interference components for IBO of $5 \mathrm{~dB}$. These are obtained through the evaluation of the derived equations and include SINR of (6) (FullDuplex SINR), ratio of Signal to Non-linear interference (SNLR) obtained as $\frac{E\left[\left|I_{d e s}\right|^{2}\right]}{E\left[\left|I_{N L}\right|^{2}\right]}$, ratio of Signal to the Self-Interference (SSIR) obtained as $\frac{E\left[\left|I_{d e s}\right|^{2}\right]}{E\left[\left|I_{S I}\right|^{2}\right]}$, ratio of Signal to the SI-plus-Full-Duplex noise (SSINR) obtained as $\frac{E\left[\left|I_{\text {des }}\right|^{2}\right]}{E\left[\left|I_{S I}\right|^{2}\right]+E\left[\left|I_{\gamma}\right|^{2}\right]}$ and finally the Signal to noise ratio at the receiver (RxSNR) evaluated as $\frac{E\left[\left|I_{d e s}\right|^{2}\right]}{E\left[\left|I_{\nu}\right|^{2}\right]+E\left[\left|I_{\eta}\right|^{2}\right]}$. Also plotted is the SINR of the traditional half-duplex mode obtained by letting $h_{S S}=0$ in (6). These plots illustrate the interplay of the various interference components; at low $\frac{\left|h_{S S}\right|}{\left|h_{G S}\right|}$ the non-linearities are 
dominant in the full-duplex mode, while the SI becomes dominant at higher values. It should also be noted that the curves for SSINR and SSIR are nearly identical indicating the negligible contribution of the full-duplexing noise. The slight change in RxSNR can be attributed to the variations in $\mathrm{OBO}$ with SI channel strength.

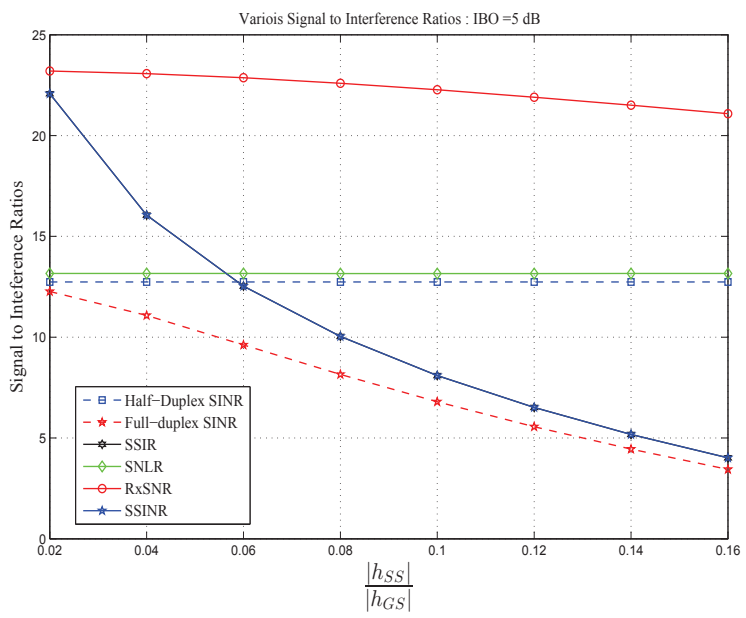

Fig. 4. Relative contribution of different interference components to SINR.

Figure 5 discusses the achieved spectral efficiency for the halfduplex and the full-duplex modes (evaluated through expressions). These curves are obtained by enumerating the spectral efficiency achievable for a given SINR using standard DVB-S2 look-up tables and dividing the result by 2 for the half-duplex case to reflect on the larger bandwidth used per path. While the rates are conservative, they indicate the dependence of spectral efficiency on the relative power levels of self-interference and the received signal. These plots can also be viewed as setting requirements on the efficiency of SI channel estimation needed to achieve gains; recall from Section III-B that, in fact, it is the channel estimation error that would be used for $h_{S S}$ instead of its actual value. As can be seen, if the SI channel (or the residual) has low power, substantial rate gain is achieved.

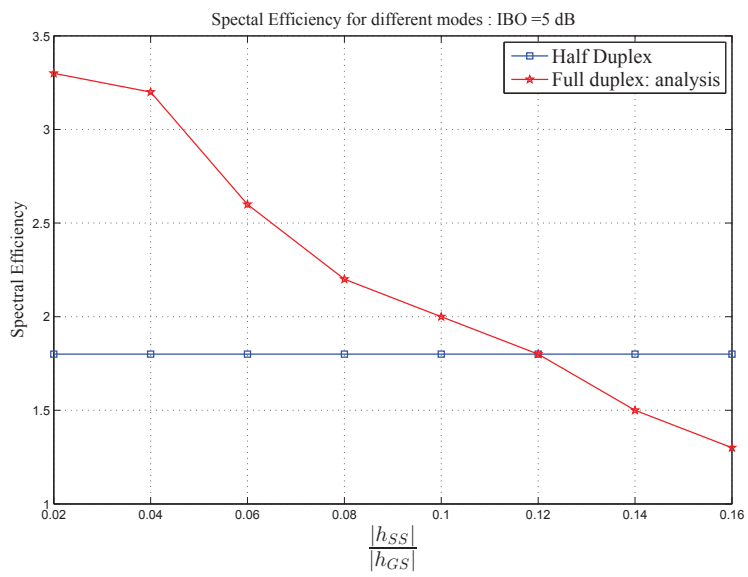

Fig. 5. Spectral Efficiency of half-duplex and full-duplex schemes $(\mathrm{IBO}=$ $5 \mathrm{~dB})$

As a final remark, thanks to the higher satellite antenna gain, it should be noted that GEO full-duplex communications is not at a particular disadvantage due to the propagation distances involved.

\section{Conclusions}

The paper explores the use of full-duplex communications in DVBS2 based satellite networks. Analysis of the various interference terms affecting the signal quality, including self-interference, nonlinear distortions and noise is performed and indications on the possible countermeasures have been provided. By comparing with the traditional half-duplex operation, it has been shown that fullduplex satellite relaying is a promising scheme enhancing the spectral efficiency under certain conditions, despite the power imbalance between the on-board transmitted and received signals. Towards realizing a full-duplex satellite system, further comprehensive studies need to be carried out to examine the feasibility of estimating the on-board self-interference channel to a high accuracy as well as the regulatory aspects towards altering existing frequency plans.

\section{REFERENCES}

[1] Digital Video Broadcasting (DVB) Second generation framing structure, channel coding and modulation systems for Broadcasting, Interactive Services, News Gathering and other broadband satellite applications (DVB-S2), ETSI EN 302 307, V1.1.2, 2006.

[2] Second generation framing structure, channel coding and modulation systems for broadcasting interactive services, news gathering, and other satellite applications, part II: S2 extensions (S2X).

[3] G.E. Corazza, "Digital Satellite Communications," Springer, 2007.

[4] G. Taricco,"Linear Precoding Methods for Multi-beam Broadband Satellite Systems", Proc. IEEE European Wireless Conference, May 2014.

[5] S. Maleki, et. al, "Cognitive Spectrum Utilization in Ka Band Multibeam Satellite Communications", IEEE Commun. Magazine, To Appear.

[6] A. Sabharwal, P. Schniter, D. Guo, D. Bliss, S. Rangarajan, and R. Wichman, "In-band full-duplex wireless: challenges and opportunities," IEEE J. Sel. Area Commun., vol. 32, no. 9, pp. 1637-1652, Sept. 2014.

[7] J. I. Choi, M. Jain, K. Srinivasan, P. Levis, and S. Katti, "Achieving single channel, full duplex wireless communication," in Proc. 16th Annual ACM International Conference on Mobile Computing and Networking, September, 2010, pp. 1-12.

[8] M. Duarte, and A. Sabharwal, "Full-duplex wireless communications using off-the-shelf radios: Feasibility and first results," in Proc. 44th Asilomar Conference on Signals, Systems, and Computers, Nov. 2010, pp. $1558-1562$.

[9] B. P. Day, A. R. Margetts, D. W. Bliss, and P. Schniter, "Full-duplex MIMO relaying: achievable rates under limited dynamic range," IEEE J. Select. Areas Commun., vol. 30, no. 8, pp. 1541-1553, Sept. 2012.

[10] E. Everett, A. Sahai, and A. Sabharwal, "Passive self-interference suppression for FD infrastructure nodes," IEEE Trans. Wireless Commun., vol. 13, no. 2, pp. 680-694, Jan. 2014

[11] M. Duarte, C. Dick, and A. Sabharwal, "Experiment-Driven Characterization of Full-Duplex Wireless Systems," IEEE Trans. Wireless Commun., vol. 11, no. 12, pp. 4296-4307, Dec. 2012.

[12] D. Bharadia, E. McMilin, and S. Katti, "Full duplex radios," in Proc. ACM Special Interest Group on Data Communication (SIGCOMM), Hong Kong, China, Aug. 2013, pp. 375-386.

[13] D. Bharadia, and S. Katti, "Full duplex MIMO radios," in 11th USENIX Symp. on Networked Systems Design and Implementation (NSDI 14), Seattle, WA, April, 2014.

[14] D. Korpi, L. Anttila, V. Syrjl, and Valkama, "Widely-linear digital selfinterference cancellation in direct-conversion full-duplex transceiver," available online: http://arxiv.org/abs/1402.6083.

[15] Paired Carrier Multiple Access (PCMA) Technology : www.viasat.com

[16] Carrier-in-Carrier (CinC) Technology : www.gilat.com

[17] S. Benedetto, E. Biglieri and R. Dafarra, "Modelling and Performance Evaluation of Nonlinear Satellite Links : A Volterra Series Approach," IEEE J. Select. Aerosp. Electron. Syst., vol. 15, pp. 494-507, July 1979.

[18] R. Piazza, M. R. Bhavani Shankar and B. Ottersten, "Data Predistortion for Multicarrier Satellite Channels Based on Direct Learning ," IEEE Trans. Signal Proc., vol. 62, no. 22, pp. 5868-5880, November 2014.

[19] R. Piazza, et. al, "Performance Analysis of Fractionally Spaced Equalization in Non-linear Multicarrier Satellite Channels," In Proc. 32nd AIAA International Communications Satellite Systems Conference, August 2014, San Diego, USA. Available online : http : //wwwen.uni.lu/snt/people/roberto_piazza. 